\title{
How do we ensure the robustness of the links between specification and evaluation in data measurement analysis and treatment?
}

\section{GEODESIGN}

How do we ensure the robustness of the links between specification and evaluation, from the product functional needs to the management of the parameters, algorithms and methods in data measurement analysis and treatment of geometrical specifications?

Today, tomorrow, the means of measurement and processing are continuously evolving.

Continuous measurements, optical scanners, X-ray vision, etc, generate increasingly larger volumes of data.

This data requires processing and a choice of method (i.e filtration) to be usable for the evaluation of dimensional and geometrical specifications

The evolution of the manufacturing and assembly processes, the reduced product and process development times require optimization of the measurements and consequently of the specifications derived from $R \& D$

All these changes, metrology means and specifications, generate an increase of the parameters and information to be taken into account in the measurement $\&$ evaluation process.

Do we have to specify all these parameters? Which ones should remain the responsibility of metrology?

How do we manage, consolidate and optimize this increasing volume of specifications \& information?

\section{Table of contents}

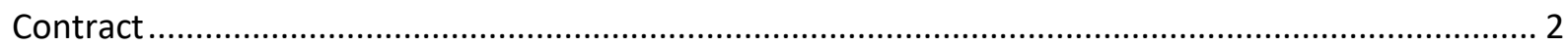

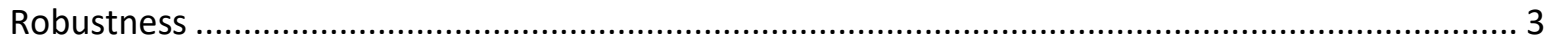

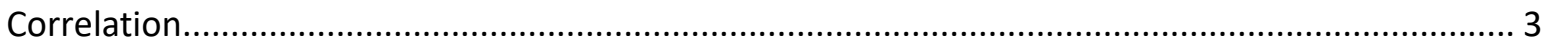

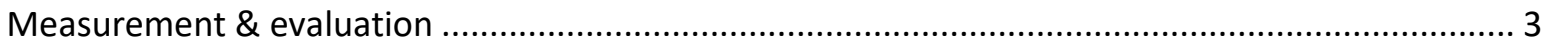

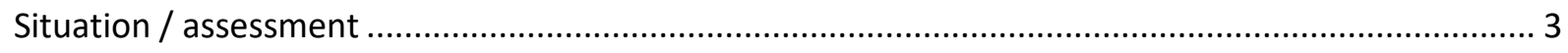

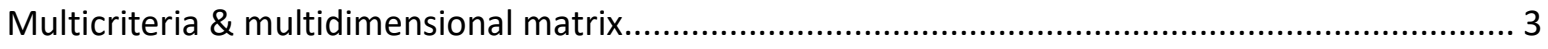

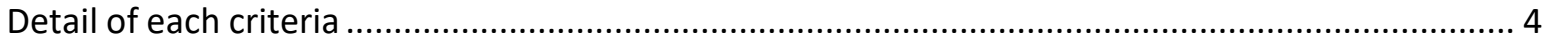

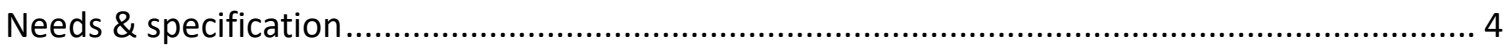

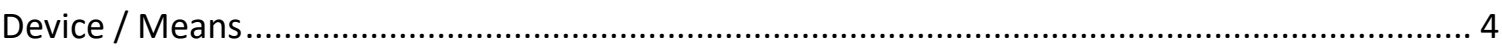

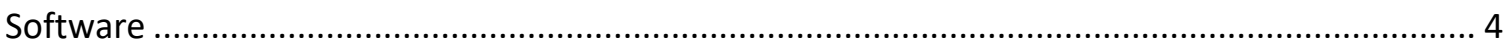

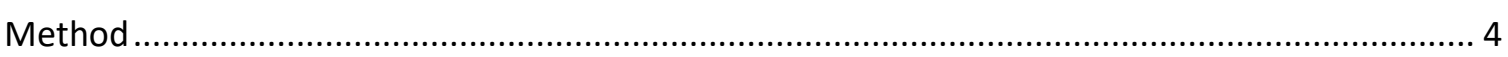

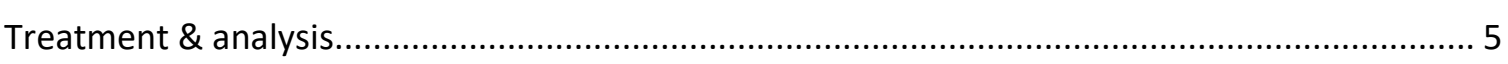

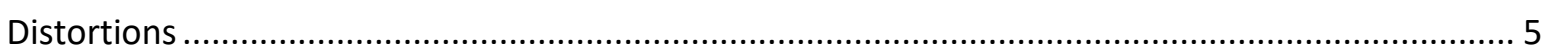




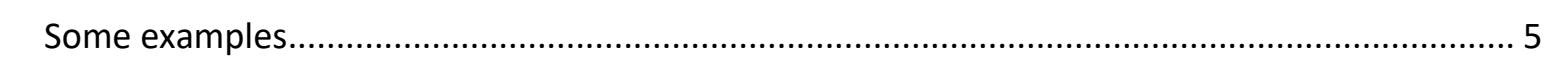

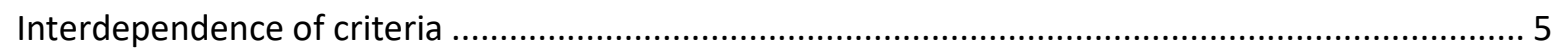

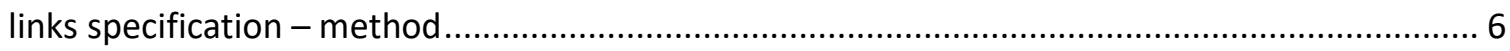

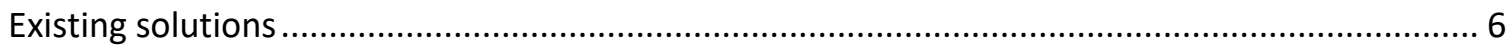

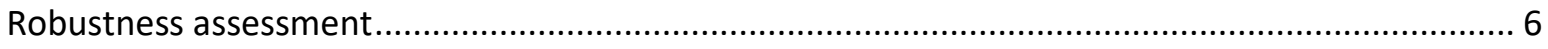

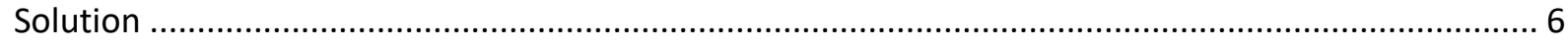

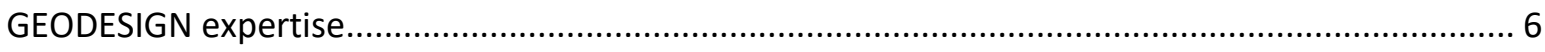

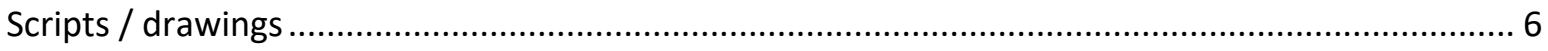

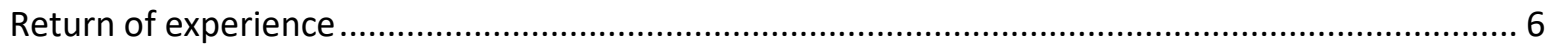

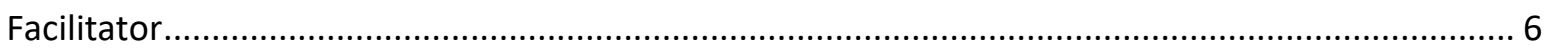

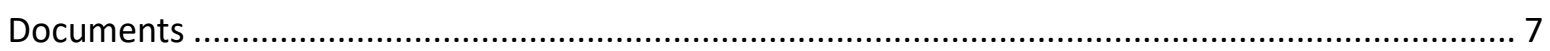

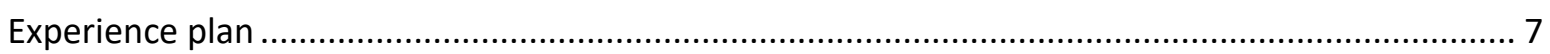

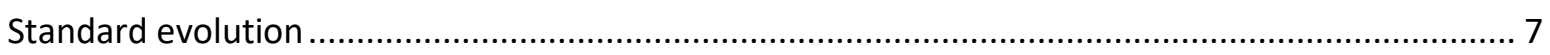

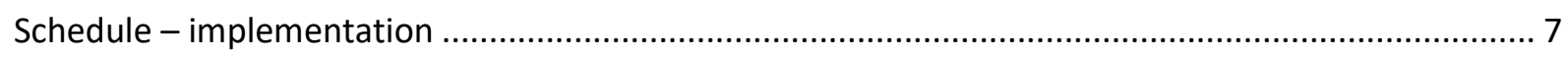

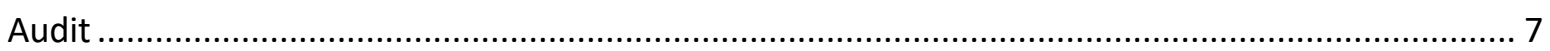

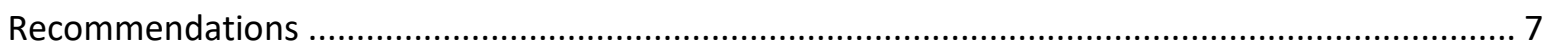

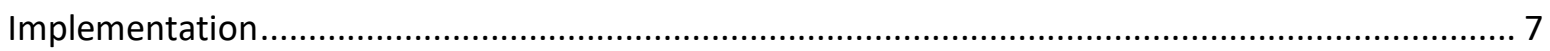

Consolidation \& improvement of the existing …................................................................ 7

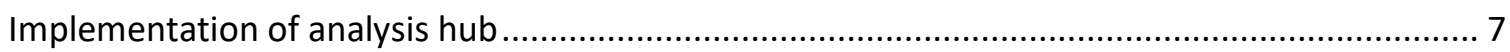

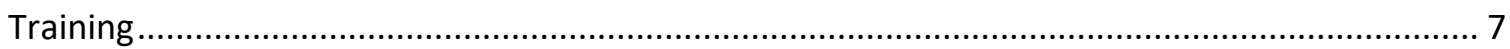

Management of liaison documents: documents and analysis file creation............................. 7

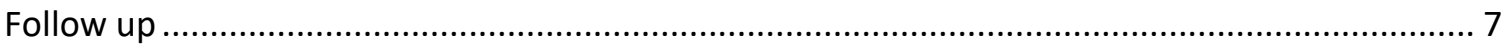

\section{Contract}

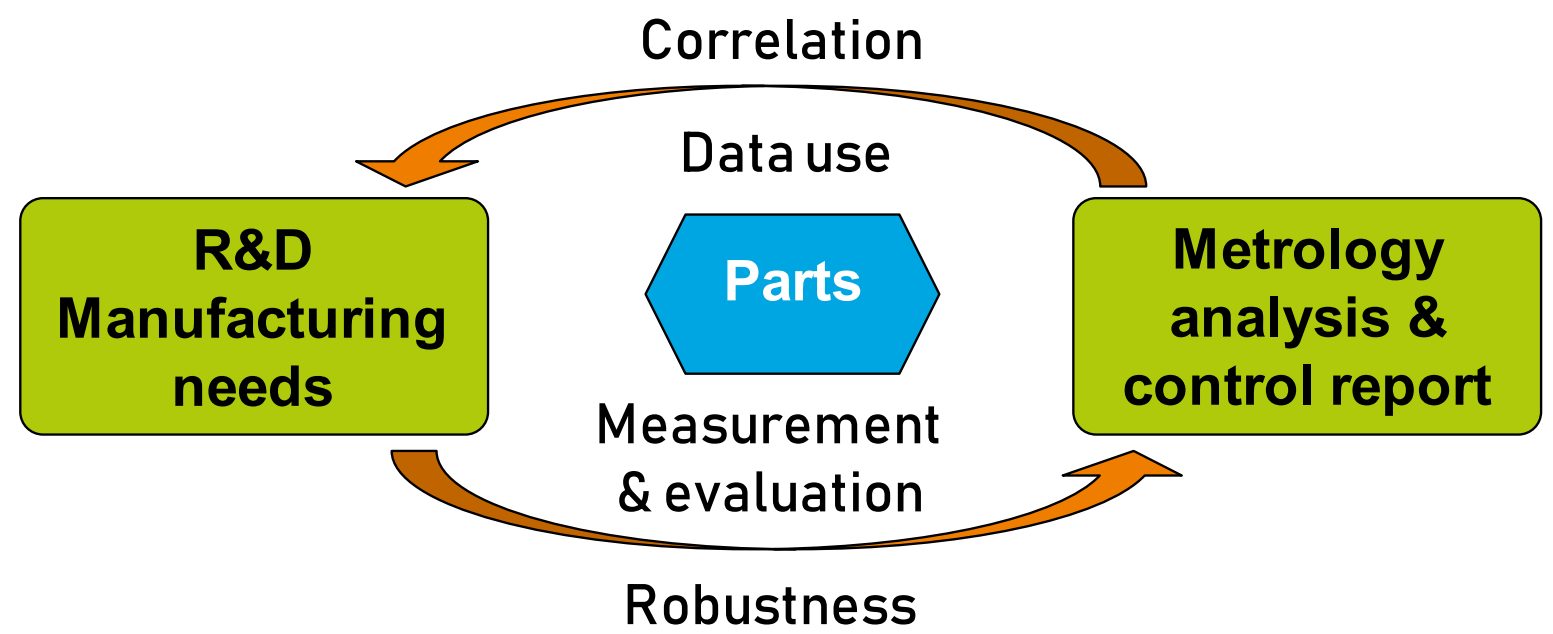




\section{Robustness}

The robustness of measurement $\&$ evaluation must be demonstrable. It takes into account the uncertainty of measuring devices and the bias generated by the translation of needs, measurements, the data treatment, the final analysis and transcription.

This is a major issue for dimensional and geometrical measurement. Results have to be published and we must be able to measure the overall uncertainty of the activity.

\section{Correlation}

The control report must include all the elements necessary for the operations of the report's applicant (manufacturing or R\&D), these elements must be clear, comprehensible and exploitable by nonmetrologists.

\section{Measurement \& evaluation}

The measurement $\&$ evaluation are composed of multiple phases and activities

\section{Activities}

- Needs analysis

- Specification (request writing and/or drawing)

- Request analysis

- Choice of the method for measurement \& evaluation

- Measurement

- Data treatment

- Interpretation / values evaluation

- Data analysis

- Report completion

- Capitalization

Each activity is independent and can be performed by several individuals

Those activities can be grouped in several phases

- Request: analysis and specification

- Preparation: request analysis, Choice of the method for measurement \& evaluation

- Measurement \& evaluation: Measurement, Data treatment, Interpretation / value evaluation

- Synthesis: Data analysis, Report completion, Capitalization

\section{Situation / assessment}

\section{Multicriteria \& multidimensional matrix}

Measurement and evaluation depend on several criteria:

- Specification

- Devices (means of measurement)

- Software for data treatment

- The method for measurement \& evaluation

For each of these criteria, several solutions, adjustments, tunings exist.... 
IN FACT, WE WORK IN A MULTICRITERIA \& MULTIDIMENSIONAL MATRIX

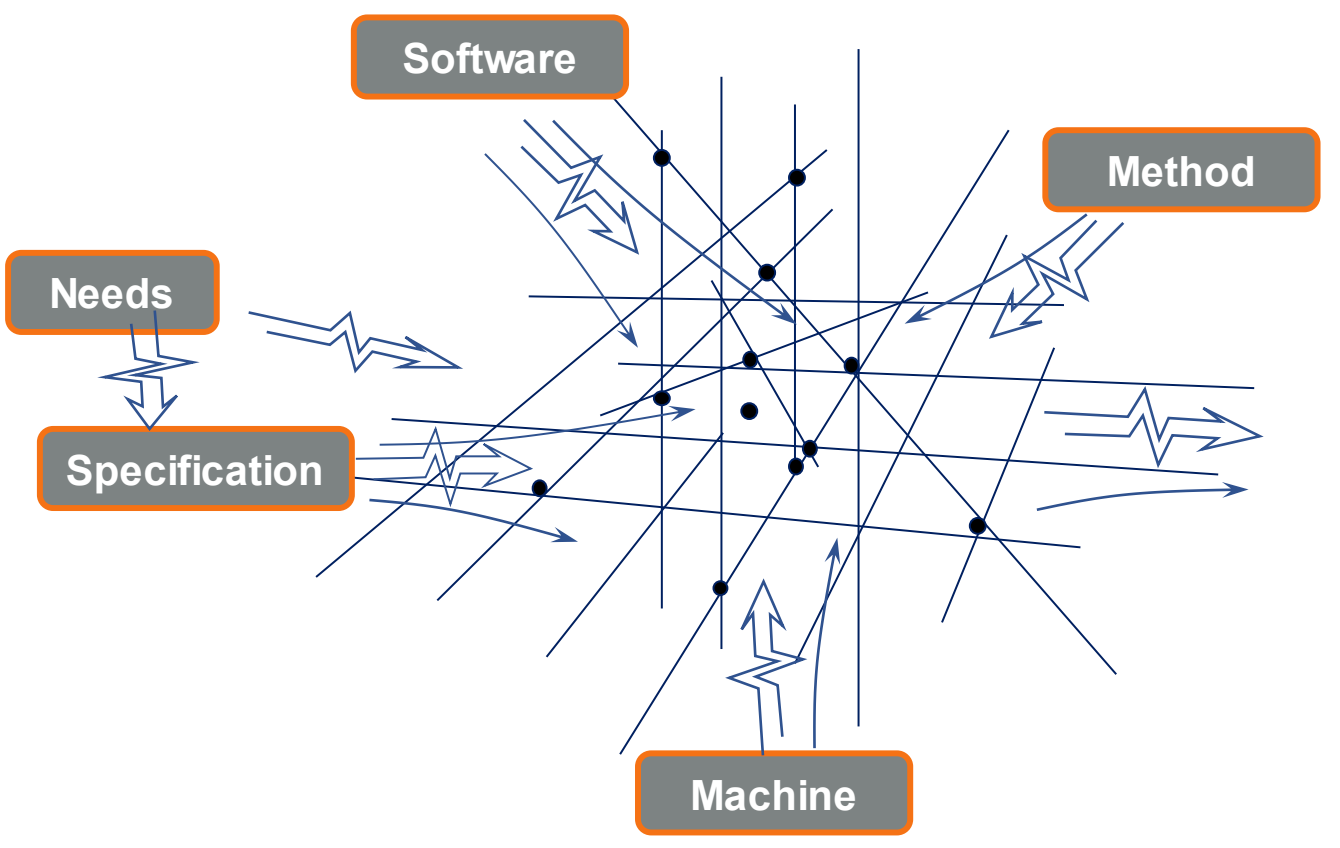

Detail of each criteria

Needs \& specification

- Datum reference system

- Geometrical and dimensional specifications

- Restrictions

- Part \& limits

- Use (function)

- Expectation (expressed or not)

Device / Means

- $\mathrm{CMM}$ contact point

- $\quad \mathrm{CMM}$ contact scanning

- Laser scanning

- Tomography

- Uncertainty

- "noise"

Software

- Algorithms

- Relation specification / treatment method

Method

- Algorithms

- Cloud of points density 
- Filtrations

- Deliverables definition

Treatment \& analysis

Each criterion, each adjustment, each choice increases the multiplicity of the cases.

This multiplicity is the major factor of the measurement diversity (i.e inter-laboratory comparison)

\section{This diversity cripples the requested robustness.}

\section{Distortions}

\section{Each parameter has its own characteristics and therefore its limits and distortions (bias)}

\section{Some examples}

- Need translation onto specifications

The ISO technical language (ISO $8015,1101 \ldots$ ) is complex and closed. It requires a deep analysis and a detailed transcription of the datum reference systems. This language is specific to the functional compliance analysis of the parts (components). It makes the transcription of other needs difficult; i.e report for tool adjustment.

Example: a wrong transcription of the datum reference systems distorts all the position evaluations

- Software

Software packages should « filter " the measured cloud of points. These filters require adjustment, of which the metrology lab is in charge. It is difficult to assess the impact of these adjustments on the accuracy of the evaluations relative to the measured part.

Example: for laser scanning, do we transform the cloud of points into a polygonal model? The "noise" will be very different

- Devices / means

A same surface measured by different types of devices (different technologies) may have divergent evaluated characteristics.

Example: Measurement with laser scanning then contact probing: the noise will be significantly different. It generates disparity

- Inter-laboratory comparison

A significant example of distortion is the inter-laboratory comparison. Considering device measurement uncertainty and a context of simple specifications, it is difficult to obtain similar (close) values for one part. The difference is often larger than the uncertainty of measurement.

Example: measurements done inside the company and the one's done in a supplier lab.

\section{Interdependence of criteria}

Finding interdependence for criteria is a difficult path. Only a few common cases can be treated

The multiplicity of criteria and their biases makes it impossible to write standard rules that cover them in their entirety. 


\section{links specification - method}

Because of the criteria multiplicity and their values, it is rare to be able to create logical links between specifications and measurement method (including value adjustments)

\section{Existing solutions}

The existing solutions, demonstrated as robust, feed the return of experience.

\section{Robustness assessment}

\section{We assess that the robustness of the link between specification and} evaluation is linked to the management of all the criteria and their distortions

\section{Solution}

\section{GEODESIGN expertise}

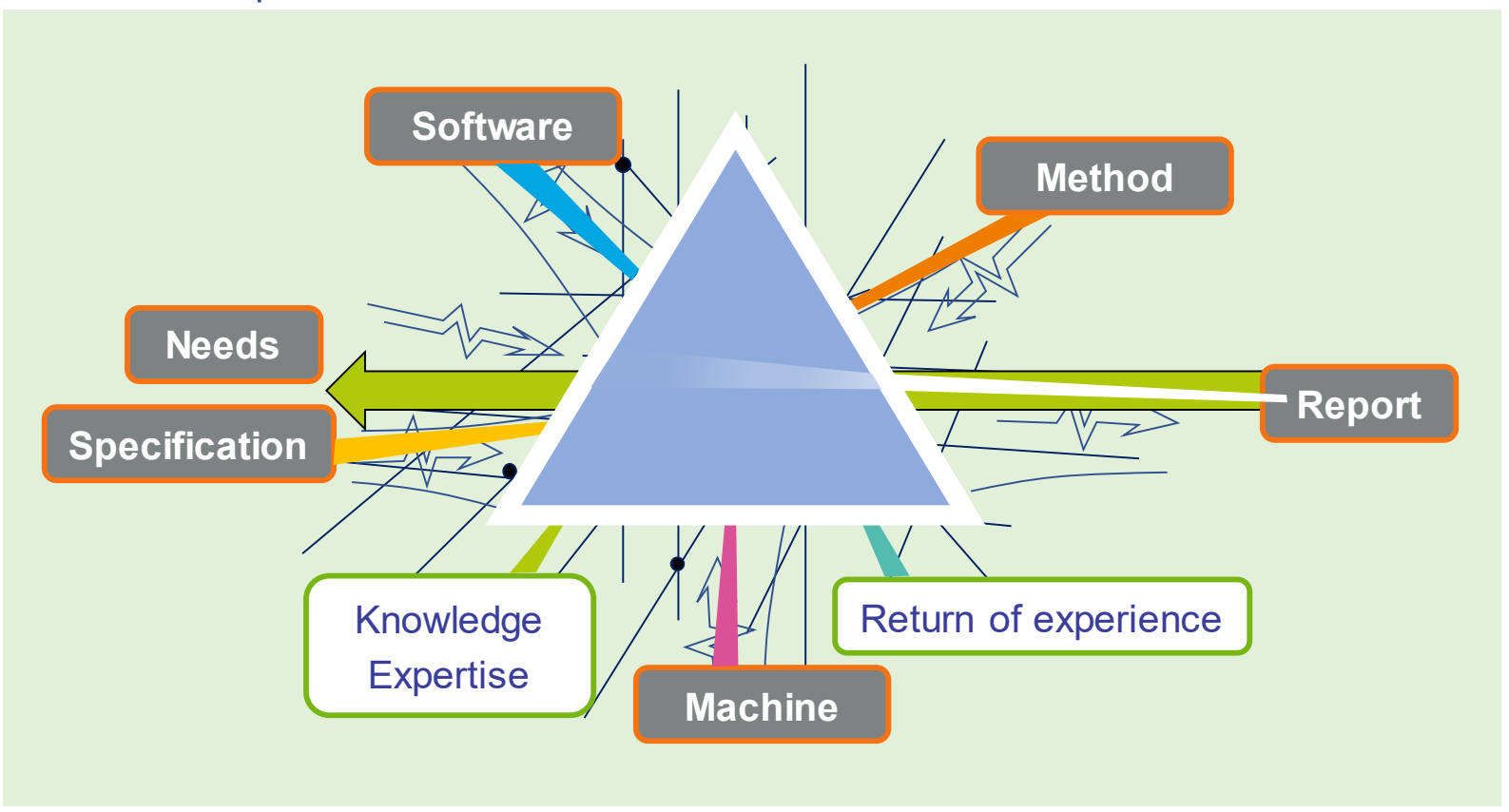

Implementing the management of the multiple parameters in order to guarantee the robustness of the link between specification and evaluation in data measurement analysis and treatment

Collecting, analysing, managing the criteria multiplicity

\section{Scripts / drawings}

The transcriptions are analysed to guarantee the link between need and specification

\section{Return of experience}

Each "solution" is analysed in order to extract a model or part of a model

Capitalisation can be in the form of libraries.

\section{Facilitator}

An analysis hub is created. Its goal is to collect, analyse and manage the different parameters \& criteria then to give recommendations for measurement \& evaluation 


\section{Documents}

The liaison documents allow to formalize the situations, to capitalize the experience and to document the measurement \& evaluation situation.

Experience plan

An experience plan can complete the scheme.

\section{Standard evolution}

ISO standards evolve all the time.

The transcription of need into specification is an axis of real improvement. Some details are difficult or impossible to translate into current technical language.

It is possible to analyze the needs and implement company-specific scripts.

Thereafter these scripts can be suggested to the standardization committee for their integration within ISO standards.

\section{Schedule-implementation}

\section{Audit}

To implement a robust solution, the starting point is an analysis, an assessment of the actual situation. This analysis includes the design office, the manufacturing and quality department and the metrology lab.

\section{Recommendations}

Depending on the objectives, the audit and the means, an implementation proposal will be delivered.

\section{Implementation}

Consolidation \& improvement of the existing

Implementation of analysis hub

Training

Management of liaison documents: documents and analysis file creation.

Follow up

\section{Hub implementation, steering \& management, training of the persons involved, expertise, consulting.}

\section{GED)ESIGN}

\section{Your partner in performance improvement www.geodesign-xyz}

